\title{
Role of Ultrastructural Alterations of Intercellular Junction and Tight-junction Proteins in Patients With Gastroesophageal Reflux Disease
}

TO THE EDITOR: I read with great interest the study by Liu et $\mathrm{al}^{1}$ regarding the significant correlation between presence of desmosomal junction alterations and the severity of gastroesophageal reflux disease (GERD). The authors reported that tight-junction proteins (TJP) such as claudin-1 and claudin-2 were abundant not only in asymptomatic controls but also in patients with gastroesophageal reflux disease and, a significant intercellular space (ICS) dilatation occurred only in upper stratified squamous epithelium of patients with moderate erosive esophagitis, while there was no difference in ICS dilatation of lower basal cuboidal epithelium among different groups of patients with GERD and controls. Furthermore, this study revealed that alterations in desmosomal junctions and their processes under transmission electron microscopy increased in parallel with the severity of GERD. Esohageal mucosal ICS were frequently seen with nonerosive reflux disease (NERD) and patients with esophagitis. ${ }^{2}$ In addition, dilatation of intercellular spaces was an early marker of damage caused by gastroesophageal reflux, and treatment of proton pump inhibitor led to a complete recovery of dilated intercellular spaces in $97.4 \%{ }^{3}$

Although the authors showed an important results of ultrastructural alterations of esophageal mucosa in patients with GERD, there were a few limitations most of which had been discussed in this paper. First, in contrast to this study, previous studies demonstrated that dilated ICS was associated with NERD and erosive GERD. For instance, Caviglia et $\mathrm{al}^{4}$ reported that mean ICS diameter in NERD patients was 3 times greater than in controls. Moreover, Zhong et $\mathrm{al}^{5}$ reported that reflux esophagitis (RE) patients showed wider ICS $(1.29 \mu \mathrm{m}$ [1.10-1.46 $\mu \mathrm{m}], \mathrm{n}=6)$ than NERD patients $(1.10 \mu \mathrm{m}$ $[0.95-1.21 \mu \mathrm{m}], \mathrm{n}=16)$ and controls $(1.01 \mu \mathrm{m}[0.94-1.17$ $\mu \mathrm{m}], \mathrm{n}=12$ ). Differences in the study population and definition of GERD severity may contribute to this discrepancy. In this study, authors defined mild RE in case of Los Angeles classi- fication A (LA-A) esophagitis and moderate RE in case of LA-B esohagitis, but there were no patients with LA-C or LA-D eosphagitis. However, Zhong et $\mathrm{al}^{5}$ classified LA-A/B into mild $\mathrm{RE}$ and LA-C/D into severe RE. I wonder whether the severe GERD patients including LA-C/D may have influence on the results or not, but there was no explanation regarding this matter in the discussion.

Second, the authors showed that there were no differences in the presence of claudin-1 and claudin-2 expression among patients with GERD, NERD and controls. However, previous study revealed that claudin-1 expression in RE and NERD patients were significantly higher than in the control. Moreover, expression of occludin tended to be lower in the RE patients and the expression of claudin-3 tended to be higher in the RE and NERD patients, however, none of these were statistically significant. ${ }^{3}$ These findings suggested the role of several other TJP including several types of claudins, occludins and junctional adhesion molecules, as authors have mentioned in the discussion.

Despite these limitations of the present study, it provides us with the important information in the ultrastructural alterations of ICS and TJP in the inflamed esophageal mucosa. Further studies are needed to validate the results of the present study.

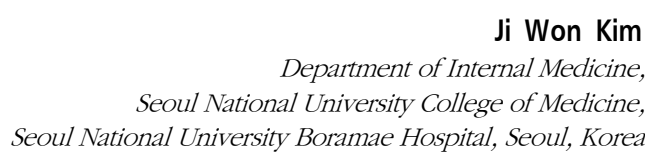

1. Liu CC, Lee JW, Liu TT, Yi CH, Chen CL. Relevance of ultrastructural alterations of intercellular junction morphology in inflamed human esophagus. J Neurogastroenterol Motil 2013;19:324-331.

2. Van Malenstein H, Farré R, Sifrim D. Esophageal dilated intercellular spaces (DIS) and nonerosive reflux disease. Am J Gastroenterol 2008;103:1021-1028.

3. Calabrese C, Bortolotti M, Fabbri A, et al. Reversibility of GERD ultrastructural alterations and relief of symptoms after omeprazole 
treatment. Am J Gastroenterol 2005;100:537-542.

4. Caviglia R, Ribolsi M, Maggiano N, et al. Dilated intercellular spaces of esophageal epithelium in nonerosive reflux disease patients with physiological esophageal acid exposure. Am J Gastroenterol 2005;100: 543-548.

5. Zhong C, Duan L, Wang K, et al. Esophageal intraluminal baseline impedance is associated with severity of acid reflux and epithelial struc- tural abnormalities in patients with gastroesophageal reflux disease. J Gastroenterol 2013;48:601-610.

\section{Conflicts of interest: None.}

